\title{
Evaluation of anti-SARS-CoV-2 antibody levels: two different methods
}

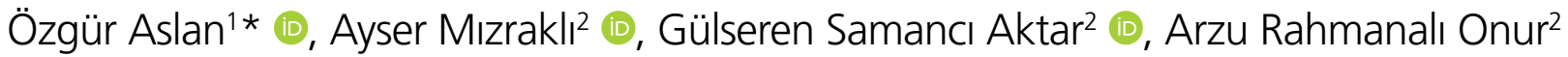

\section{SUMMARY}

OBJECTIVE: Severe acute respiratory syndrome coronavirus 2 (SARS-CoV-2) disease (COVID-19) is still a major problem worldwide. Antibody response to SARS-CoV-2 has not yet been fully clarified, and clinical benefits of serological tests remain unclear. Despite the presence of numerous systems and methods used to analyze antibody levels, it is difficult to mention about standardization. This study aims to evaluate antibody levels of COVID-19 patients obtained by different methods.

METHODS: Specimens of 55 patients were included in this study. Patients underwent SARS-CoV-2 real-time polymerase chain reaction test, COVID-19 IgM/lgG antibody rapid test (Hotgen), and Roche SARS-CoV-2 antibody test.

RESULTS: In this study, the positive values of COVID-19 IgM/lgG antibody rapid test, Roche SARS-CoV-2 antibody test, and SARS-CoV-2 real-time polymerase chain reaction test were 37,26 , and 31 , respectively, whereas the negative values were 18,29 , and 24 , respectively. A comparison of the results using $\chi^{2}$ test revealed a significant difference among SARS-CoV-2 real-time polymerase chain reaction, COVID-19 IgM/lgG antibody rapid test (Hotgen), and Roche SARS-CoV-2 antibody test.

CONCLUSIONS: We recommend antibody testing in close contact tracing as well as in real-time polymerase chain reaction negative symptomatic subjects. Standardization is important as positive values show significant variations among antibody tests.

KEYWORDS: SARS-CoV-2. Immunoassay. Serological tests. Immunoglobulins.

\section{INTRODUCTION}

Severe acute respiratory syndrome coronavirus 2 (SARSCoV-2) disease (COVID-19) is still a major problem all over the world ${ }^{1,2}$. A substantial number of patients and deaths due to SARS-CoV-2 has been achieved worldwide, and unfortunately the number of cases continues to increase ${ }^{3}$. Accurate and rapid diagnosis of SARS-CoV-2 is essential for timely isolation of COVID-19 patients to stop the pandemic and to save the people's lives. The real-time polymerase chain reaction (RT-PCR) test, which has been developed and used for rapid detection of SARS-CoV-2, is used to detect viral nucleic acid and as the standard diagnostic test for COVID-19. Being time-consuming and troublesome and requiring specific equipment have restricted the use of RT-PCR test particularly in the areas with limited laboratory facilities ${ }^{2,4}$.

Human antibody response against viral infection has been widely used to help with the diagnosis of viral infections. Comparing with the RT-PCR tests, the detection of antibody levels is more easily accessible as these tests are faster, cheaper, easy to use, and less frequently require laboratory expertise ${ }^{2}$. Antibody reactions against SARS-CoV-2 remain unclear, and the clinical benefits of serological tests are indefinite ${ }^{5}$.

Long et al. ${ }^{5}$ collected the serum samples from 164 subjects for antibody testing approximately after 30 days of exposure

\footnotetext{
${ }^{1}$ Health Sciences University Gazi Yaşargil Training and Research Hospital, Department of Clinical Chemistry Laboratory - Kayapınar, Turkey. ${ }^{2}$ Health Sciences University Gazi Yaşargil Training and Research Hospital, Department of Clinical Microbiology Laboratory - Kayapınar, Turkey. *Corresponding author: ozguraslan44@yahoo.com Conflicts of interest: the authors declare there are no conflicts of interest. Funding: none. Received on December 30, 2020. Accepted on January 09, 2021.
} 
to the virus. Virus-specific IgG and/or IgM were found positive in all of 16 cases with positive RT-PCR result. In addition, virus-specific IgG and/or IgM were also found positive in seven out of 148 subjects with negative RT-PCR, indicating that $4.3 \%$ (7/164) of close contacts have been missed out by the RT-PCR test ${ }^{5}$. Seroconversion for IgM is achieved in five days after symptom onset, whereas seroconversion for $\mathrm{IgG}$ is achieved in 5-7 days after symptom onset ${ }^{6,7}$. Maximum seroconversion occurs in 2-3 weeks for IgM and in 3-6 weeks for $\mathrm{IgG}^{5-7}$. It is known that studies about this test are lacking or limited in number as this is a novel test.

The data from SARS pandemic indicate that serological responses including virus-specific $\operatorname{IgM}$ and $\operatorname{IgG}$ are adequate for making serological diagnosis ${ }^{8,9}$. The results of these studies revealed that the ELISA tests require less labor for serological diagnosis of SARS-CoV-2 pneumonia and are sensitive, more economic, and provide an advantage as they do not require viral cultivation?.

The rapid test uses a double-antigen sandwich method to detect SARS-CoV-2 IgM/IgG antibodies and is a colloidal gold-based immunochromatographic assay ${ }^{10}$. The Roche AntiSARS-CoV-2 assay is an immunologic test for in vitro quantitative detection of SARS-CoV-2 antibodies (including $\mathrm{IgG}$ ) in human serum and plasma. It was aimed to use this test as an assistant in detecting immune response against SARS-CoV-2. The Roche Elecsys Anti-SARS-CoV-2 assay uses a recombinant protein for nucleocapsid antigen to detect SARS-CoV-2 antibodies ${ }^{11}$.

Despite the presence of numerous antibody tests concerning COVID-19 pandemic, reliability or specificity comparisons have not been performed for many of them. The antibody testing will become more important with the increasing number of individuals affected by the pandemic. This study aimed to evaluate the results of the COVID-19 IgM/IgG rapid test (Pekin Hotgen Biotech Co., Ltd.) used in our hospital with the results of the Roche SARS-CoV-2 (Roche Diagnostics, Germany) assay studied in immunoassay system.

\section{METHODS}

Specimens from a total of 55 patients that have applied to our hospital between April and May 2020 were included in this study. All patients underwent the SARS-CoV-2 RT-PCR testing (Bioeksen, Turkey) of nasopharyngeal smear as well as the COVID-19 IgM/IgG rapid testing and the Roche SARS$\mathrm{CoV}-2$ antibody testing of blood samples at least once. For all the antibody testing procedures, the blood samples were collected into routine tubes, waited for $30 \mathrm{~min}$ for clotting to occur, centrifuged at $1500^{\prime} \mathrm{g}$ and then stored at $-70^{\circ} \mathrm{C}$ until the time of analysis. Completely resolved serum specimens were studied.

Of the 55 individuals from whom the study samples have been obtained, 32 were females and 23 were males. The age of these individuals ranged between 21 and 91 years, with a mean age of $33.96 \pm 11.849$ years.

The Bio-Speedy Direct RT-qPCR SARS-CoV-2 nucleic acid detection kit (Bioeksen, Turkey) is designed for the qualitative detection of nucleic acid from SARS-CoV-2. The kit is a one-step reverse-transcription and real-time PCR assay targeting the SARS-CoV-2-specific $\mathrm{N}$ and ORF1ab gene region. Nasopharyngeal swab samples collected in viral nucleic acidbuffered tubes for SARS-CoV-2 RT-PCR were analyzed by using the Bio-Rad CFX96 Touch Thermal Cycler (Bio-Rad Laboratories, Hercules, CA, USA) device. RT-PCR was performed at $52^{\circ} \mathrm{C}$ for five min ( 1 cycle), $95^{\circ} \mathrm{C}$ for $10 \mathrm{sec}$ ( 1 cycle) followed by $95^{\circ} \mathrm{C}$ for one sec and $55^{\circ} \mathrm{C}$ for $30 \mathrm{sec}$ ( 40 cycles) steps ${ }^{12}$. The negative and positive controls for each study and the internal controls for each sample were evaluated, and the PCR result was interpreted as positive or negative result based on the appropriate controls.

All samples were analyzed by the COVID-19 IgM/IgG rapid testing, as well as with the original kits after the twophase quality-control procedure in the Roche cobas e 601 device (Roche Diagnostics, Germany), which is routinely used in our laboratory.

The Roche SARS-CoV-2 antibody test is a test based on chemiluminescent immunoassay. The results are determined automatically by the software, comparing the electrochemiluminescence signal obtained from the reaction product with the signal of the threshold value previously obtained by calibration. The results of the samples analyzed in Roche cobas e 601 device are presented as reactive or unreactive and as cut-off index (COI). $\mathrm{COI}<1.0$ nonreactive is interpreted as negative for Anti-SARS-CoV-2 antibody, COI $\geq 1.0$ reactive is interpreted as positive for Anti-SARS-CoV-2 antibody ${ }^{11}$. The data were compared using the $\chi^{2}$ test.

\section{RESULTS}

Specimens from a total of 55 individuals were included in this study. All patients underwent the SARS-CoV-2 RT-PCR, the COVID-19 IgM/IgG rapid testing, and the Roche Anti-SARS$\mathrm{CoV}-2$ antibody testing procedures. The number of test-positive patients was 31,37 , and 26, respectively, and the number of test-negative patients was 24,18 , and 29 , respectively, for each method (Table 1).

Among 37 patients with positive rapid antibody test result, seven patients had negative SARS-CoV-2 RT-PCR test result 
Table 1. Number of patients.

\begin{tabular}{l|c|c}
\multirow{2}{*}{ Method } & \multicolumn{2}{|c}{$\mathrm{n}=55$} \\
\cline { 2 - 3 } & Positive & Negative \\
\hline COVID-19 IgM/lgG rapid test & 37 & 18 \\
\hline Roche Anti-SARS-CoV-2 & 26 & 29 \\
\hline SARS-CoV-2 RT-PCR & 31 & 24 \\
\hline
\end{tabular}

and 11 had negative Roche Anti-SARS-CoV-2 antibody test result. Only one out of the 11 patients with negative Roche Anti-SARS-CoV-2 antibody test result had negative SARSCoV-2 RT-PCR test result.

While the Roche Anti-SARS-CoV-2 antibody test result was negative in 18 patients with positive rapid antibody test result, only one patient had positive SARS-CoV-2 RT-PCR test result. Overall, the Roche Anti-SARS-CoV-2 antibody test result was positive in 26 and negative in 29 patients.

The consistency between the SARS-CoV-2 RT-PCR test and the Roche SARS-CoV-2 assay was $76.9 \%$ for positive patients and $62.1 \%$ for negative patients, and the difference is significant $\left(\chi^{2}=8.47, \mathrm{p}<0.004\right)$. The consistency between the SARS-CoV-2 RT-PCR test and the COVID-19 IgM/IgG rapid test was $81.1 \%$ for positive patients and $94.4 \%$ for negative patients, and the difference is significant $\left(\chi^{2}=28.08, \mathrm{p}<0.001\right)$. The consistency between the COVID-19 IgM/IgG rapid test and the Roche SARS-CoV-2 assay was $70.3 \%$ for positive patients and $100 \%$ for negative patients, and the difference was significant $\left(\chi^{2}=23.99, \mathrm{p}<0.001\right)$.

\section{DISCUSSION}

This study was carried out with the specimens obtained from 55 patients in our hospital. The RT-PCR test is used for rapid detection of SARS-CoV-2. The studies about the occurrence of antibody response following SARS-CoV-2 transmission and the stability of antibodies are limited. Although antibody testing using quick tests is older than antibody testing in immunoassay systems, comparative studies in the literature are lacking due to small number of the studies on this subject. Both the COVID-19 IgM/IgG rapid test and the Roche SARS-CoV-2 assay determine the total antibody level, but do not discriminate virus-specific IgM from IgG.

Zhao et al. ${ }^{13}$ conducted a study in 173 patients and found seroconversion rate of $93.1 \%$ for overall antibodies, $82.7 \%$ for $\operatorname{IgM}$, and $64.7 \%$ for $\operatorname{IgG}$. They reported that antibody negativity determined in 12 patients might have been resulted from not analyzing the specimens in the advanced stages of the disease ${ }^{13}$. In this study, the antibody tests gave negative results but the PCR test gave positive result in a single case, although there were seven days between PCR and antibody testing procedures.
Among seven patients who were positive for the COVID$19 \mathrm{IgM} / \mathrm{IgG}$ rapid test but negative for the RT-PCR test, specimen for the RT-PCR testing was obtained on the same day from two patients, both on the same day and before 20,38, and 40 days, respectively, from three patients, and before 42 and 60 days from two patients. The Roche SARS-CoV-2 antibody test result was positive in six of the seven patients with positive COVID-19 IgM/IgG rapid test result. Wang et al. ${ }^{14}$ found the positivity rates for SARS-CoV-2 RNA of 63\% in nasopharyngeal smear specimens and 32\% in oropharyngeal smear specimens $^{14}$. RT-PCR negativity in seven patients $(12.72 \%)$ with antibody positive can be attributed to specimen-related positivity or time of sample collection. Accordingly, it can be concluded that antibody testing in symptomatic patients negative for RT-PCR is beneficial in identifying these patients. The lower number of RT-PCR positivity than antibody positivity in this study might be associated with all the specimens being nasopharyngeal smear because RT-PCR positivity is higher with the specimens obtained from lower respiratory tract such as bronchoalveolar lavage fluid and deep tracheal aspirate. Moreover, PCR positivity is associated with numerous factors including RT-PCR performance, quality and consistency of the PCR kits used, sample collection skills, and type of samples ${ }^{13,14}$.

The detection of antibody levels is critical for making a diagnosis in the patients with negative RT-PCR test result; antibody-positive healthy individuals who are in quarantine period because of close contact should be considered as potential carriers and should undergo the RT-PCR testing more frequently, and antibodies detected in RT-PCR-positive patients indicate induction of specific antibodies in the individuals ${ }^{13}$. Detecting higher antibody positivity when compared with RT-PCR test positivity indicates that antibody testing can be used not to miss the cases and to take necessary isolation measures during pandemic. Besides, antibody testing may help with diagnosis because symptomatic and RT-PCR-negative patients with low viral burden might be overlooked. Long et al. ${ }^{5}$ studied 16 specimens (i.e., three from asymptomatic patients) from 164 close contacts and determined RT-PCR positivity as well as virus-specific IgM and/or IgG seropositivity; however, they determined RT-PCR negativity and virus-specific IgM and/or IgG seropositivity in the specimens from seven of the remaining 148 asymptomatic patients ${ }^{5}$. In this study, there was only one patient (1.81\%) with the specimen positive for RT-PCR and negative for antibody testing among 55 specimens, whereas seven (12.72\%) specimens showed positivity for rapid antibody testing and negativity for RT-PCR. In immunoassay system, however, RT-PCR was negative in three patients with antibody positive. The rapid test was not negative in any of the Roche Anti-SARS-CoV-2 antibody-positive specimens analyzed by immunoassay system. Among the antibody 
tests in this study, rapid test has higher antibody positivity rates (e.g., antibody positivity rate $67.27 \%$ for rapid test and $52.72 \%$ for immunoassay test). The rapid test and the immunoassay test differ significantly from each other in terms of detecting AntiSARS-CoV- 2 antibodies $\left(\chi^{2}=23.99, \mathrm{p}<0.001\right)$.

In the study carried out using 208 plasma specimens (i.e., from 82 confirmed and 58 asymptomatic PCR-negative patients, 140 in total), Guo et al. ${ }^{15}$ reported that the efficacy of IgM ELISA is higher than PCR after 5.5 days of symptom onset and that the positivity rate increases significantly with IgM ELISA plus PCR (98.6\%) when compared with PCR alone $(519 \%)^{15}$. Many studies have emphasized that serological tests can increase the positivity rate and that they should be used in subclinical patients and, in future, epidemiological studies ${ }^{16-18}$. Also, in this study, considering the consistency between the tests in terms of both positivity and negativity rates, we concluded that antibody testing is important in the diagnosis and patient monitoring.

We reached to seven patients (hospital staff) with antibody positive and RT-PCR negative determined in this study; these patients confirmed that they have had COVID-19 and have undergone antibody testing accordingly. Therefore, it can be concluded that rapid test positivity is not a false positivity. In addition, the significant difference between the Anti-SARS-CoV-2 antibody test and the SARS-CoV-2 RT-PCR test indicates the importance of antibody testing. In general, although immunoassay systems show better sensitivity and specificity, one of the striking outcomes of this study is higher antibody positivity rate with rapid test when compared with the immunoassay system. Therefore, studies on antibody levels gain importance in determining the seroprevalence among population and in detecting antibody levels for both diagnostic and therapeutic purposes.

\section{CONCLUSIONS}

The antibody testing might be important for close contact tracing. Moreover, we believed that antibody testing should be performed in RT-PCR-negative symptomatic patients. In addition, since the positivity rate shows significant difference among antibody tests as mentioned in this study, studies on this subject and standardization are of importance.

\section{ACKNOWLEDGMENTS}

The authors thank Diyar-Med Health Products Ind. and Com. Ltd. Co. for supplying the Roche SARS-CoV-2 antibody test kit.

\section{ETHICAL APPROVAL}

This study was approved by the Ethics Committee of Health Sciences University Diyarbakır Gazi Yasargil Training and Research Hospital (dated October 16, 2020, Decision No. 601 , including reference number).

\section{AUTHORS" CONTRIBUTIONS}

OA: Conceptualization, Data Curation, Formal Analysis, Funding Acquisition, Investigation, Methodology, Project Administration, Resources, Software, Supervision, Validation, Visualization, Writing - Original Draft, Writing - Review \& Editing. AM: Data Curation, Investigation, Supervision, Visualization, Writing - Review \& Editing. GSA: Formal Analysis, Methodology, Resources, Supervision, Validation, Visualization, Writing - Review \& Editing. ARO: Data Curation, supervision, Supervision, Visualization, Writing Review \& Editing.

\section{REFERENCES}

1. Singhal, T. A review of coronavirus disease-2019 (COVID-19). Indian J Pediatr. 2020(87): 281-6. https://doi.org/10.1007/ s12098-020-03263-6

2. Xiang F, Wang $X$, He X, Peng Z, Yang B, Zhang J, et al. Antibody detection and dynamic characteristics in patients with coronavirus disease 2019. Clin Infect Dis. 2020;71(8):1930-4. https://doi.org/10.1093/cid/ciaa461

3. World Health Organization. WHO Coronavirus Disease (COVID19) Dashboard 2020. Geneva: World Health Organization; 2020. [cited on Dec. 5, 2020]. Available from: https://covid19.who.int/

4. Tang YW, Schmitz JE, Persing DH, Stratton CW. Laboratory diagnosis of COVID-19: current issues and challenges. J Clin Microbiol. 2020;58(6):e00512-20. https://doi.org/10.1128/ JCM.00512-20

6. Long QX, Liu BZ, Deng HJ, Wu GC, Deng K, Chen YK, et al. Antibody responses to SARS-CoV-2 in patients with COVID-
19. Nat Med. 2020;26(6):845-8. https://doi.org/10.1038/ s41591-020-0897-1

7. Liu W, Liu L, Kou G, Zheng Y, Ding Y, Ni W, et al. Evaluation of nucleocapsid and spike protein-based enzyme-linked immunosorbent assays for detecting antibodies against SARS-CoV-2. J Clin Microbiol. 2020;58(6):e00461-20. https:// doi.org/10.1128/JCM.00461-20

8. To KKW, Tsang OTY, Leung WS, Tam AR, Wu TC, Lung DC, et al. Temporal profiles of viral load in posterior oropharyngeal saliva samples and serum antibody responses during infection by SARS-CoV-2: an observational cohort study. Lancet Infect Dis. 2020;20(5):565-74. https://doi.org/10.1016/S14733099(20)30196-1

9. Louie JK, Hacker JK, Mark J, Gavali SS, Yagi S, Espinosa A, et al. SARS and common viral infections. Emerg Infect Dis. 2004;10(6):1143-6. https://doi.org/10.3201/eid1006.030863 
10. Woo PC, Lau SK, Wong BH, Tsoi HW, Fung AM, Chan KH, et al. Detection of specific antibodies to severe acute respiratory syndrome (SARS) coronavirus nucleocapsid protein for serodiagnosis of SARS coronavirus pneumonia. J Clin Microbiol. 2004;42(5):2306-9. https://doi.org/10.1128/JCM.42.5.2306-2309.2004

11. Beijing Hotgen Biotech Co.,Ltd. [editor]. Coronavirus disease (COVID 19) IgM/lgG antibody rapid test (Colloidal Gold) kit prospectus. Beijing: Beijing Hotgen Biotechn Co., Ltd.; 2020.

12. Roche [editor]. Elecsys Anti-SARS-CoV-2 S. Mannheim: Roche; 2020. [cited on Dec. 3, 2020]. Available from: https:// pim-eservices.roche.com/eLD/api/downloads/91e382bc-4cf6ea11-fe90-005056a772fd? countrylsoCode $=\mathrm{tr}$

13. Bioeksen. Bio-Speedy ${ }^{\circledR}$ SARS-CoV-2 (2019-nCoV) RT-qPCR. Istanbul: Bio-speedy; 2021. [cited on Dec. 3, 2020]. Available from: https://mww.bioeksen.com.tr/Media/Documents/biospeedy-sarscov2-2019ncov-rtqpcr-detection-kit-ifucdf32.pdf

14. Zhao J, Yuan Q, Wang H, Liu W, Liao X, Su Y, et al. Antibody responses to SARS-CoV-2 in patients with novel coronavirus disease 2019. Clin Infect Dis. 2020;71(16):2027-34. https:// doi.org/10.1093/cid/ciaa344
15. Wang W, Xu Y, Gao R, Lu R, Han K, Wu G, et al. Detection of SARS-CoV-2 in different types of clinical specimens. JAMA. 2020;323(18):1843-4. https://doi.org/10.1001/ jama.2020.3786

16. Guo L, Ren L, Yang S, Xiao M, Chang D, Yang F, et al. Profiling Early Humoral Response to Diagnose Novel Coronavirus Disease (COVID-19). Clin Infect Dis. 2020;71(15):778-85. https://doi. org/10.1093/cid/ciaa310

17. Zhang W, Du RH, Li B, Zheng XS, Yang XL, Hu B, et al. Molecular and serological investigation of 2019-nCoV infected patients: implication of multiple shedding routes. Emerg Microbes Infect. 2020;9(1):386-9. https://doi.org/10.1080/22221751 .2020 .1729071

18. Du Z, Zhu F, Guo F, Yang B, Wang T. Detection of antibodies against SARS-CoV-2 in patients with COVID-19. J Med Virol. 2020;92(10):1735-8. https://doi.org/10.1002/jmv.25820

19. Jin $Y$, Wang $M$, Zuo Z, Fan C, Ye F, Cai Z, et al. Diagnostic value and dynamic variance of serum antibody in coronavirus disease 2019. Int J Infect Dis. 2020;94:49-52. https://doi. org/10.1016/j.ijid.2020.03.065 